MATEC Web of Conferences 43, 03001 (2016)

DOI: $10.1051 /$ matecconf/20164303001

(C) Owned by the authors, published by EDP Sciences, 2016

\title{
High-Resolution Spectroscopy through Tunable, Ultrastable Optical Radiation Diode Laser
}

\author{
Brij Mohan Kumar Prasad ${ }^{1, a}$, Aparna Bawankan² \\ ${ }^{1}$ Dronacharya College of Engineering, Gurgaon-1225603, Haryana, India \\ ${ }^{2}$ Dronacharya College of Engineering, Gurgaon-1225603, Haryana, India
}

\begin{abstract}
We report onthe combination of good tunability with superb short-term frequency, stability and controllability which exhibits the excellent spectral properties of a diode laser set up. The concept of this is based on merging of the diode laser with resonant optical feedback and the grating stabilized diode laser. The short-term performance to characterize it is batted two essentially identical diode lasers also to find short-term line width. Between these lasers phase locking is achieved with a servo small bandwidth although it is required for subradian residual phase error to use of an analog phase detector. The concept of the laser is suitable for atomic or molecular coherence experiments only due to the excellent performance.
\end{abstract}

\section{Introduction}

A diode laser system exhibits its all the advantages of grating stabilized diode lasers. The system is good overall and continuous tunability, the reliability and simplicity of operation. Because of the spectral availability and a good overall with continuous tunability, diode lasers are well suitable for a variety of laser spectroscopic application (1). The diode lasers fabricated on an off-angle substrate with high doping has been an entrant for the red source for color laser displays (2). If relative stability of hertz is required the diode lasers exhibits broadband frequency which can pose a severe obstacle for locking diode lasers to an optical reference. An investigation through experimental layout atomic coherence or coherence between individual rotational and vibrational molecular states is required for relative phase locking of different lasers (3, 4). An optical flywheel with hertz linewidth for the realization of an optical clock is mandatory (5). If a grating diode laser is employed it typically requires a frequency control servo bandwidth of a few megahertz (6). For the excellent short-term stability and relatively small frequency noise bandwidth there is a common advantage to diode lasers with resonant optical feedback.

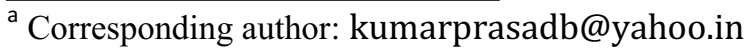

\section{Experimental setup}

Fig. 1 shows the experimental diode laser setup which is operating at $1420 \mathrm{~nm}$. Basically it consists as: D- a grating diode laser in Littman configuration; COL-Collimator; GRT-transmission grating; HWP- half-wave plate; MC- (T-8.2\%), MP, MF (both $\mathrm{R}=99.7 \%$ )- mirrors defined the external cavity CAV, HCD- balanced polarization detector is part of the internal stabilization; OD- optical diode.

The light emitted by the laser diode is diffracted by a transmission grating. With the efficiency of $95 \%$ the first diffraction is coupled into a folded cavity which provides an optical feedback to the laser diode. The optical experimental setup constitutes two coupled cavities which are defined by two mirrors MP and MC, and MF with the rear facette of the laser diode chip. To guarantee reliable single-mode operation, the coupled cavity configuration necessitates a relative frequency stabilization. By means of a polarization- sensitive stabilization scheme (7), the internal stabilization is realized and setup of mirror MP at actual position. The two essentially identical diode lasers are used all at constant temperature. The tuning is ranged continuously corresponding to up to $20 \mathrm{GHz}$. 


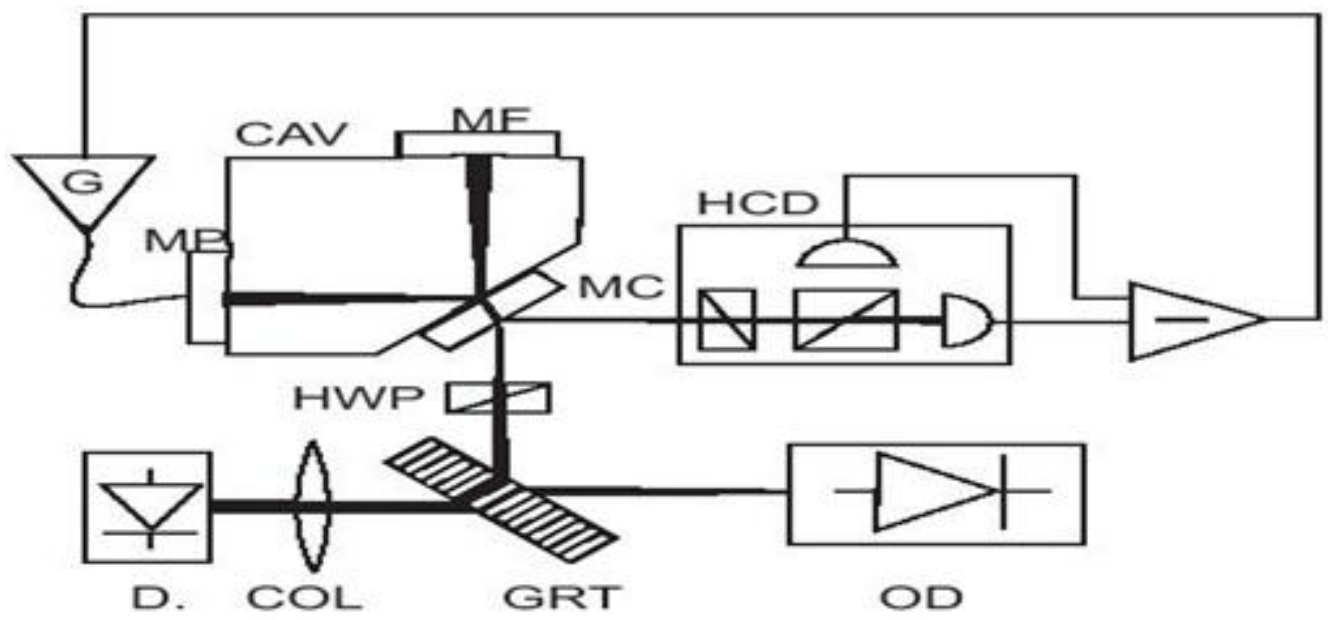

Figure 1. Experimental Setup for diode laser

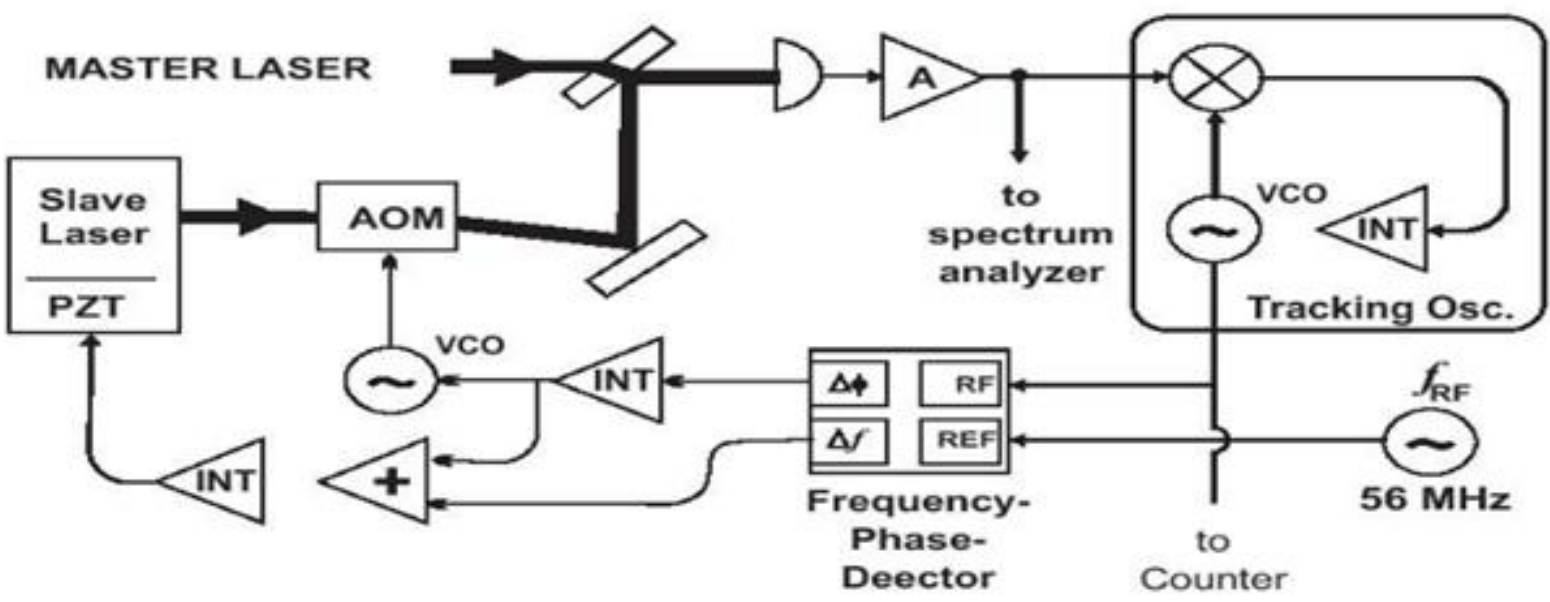

Figure 2. Investigation of the short-term frequency stability \& phase locking performanc

There was a performance of a beat-note measurement between the two lasers to demonstrate the excellent short-term frequency stability. With a servo bandwidth of $\sim 1 \mathrm{kHz}$ to eliminate low- frequency relative frequency jitter, frequency lock the lasers to each other. Fig. 2 shows experimental setup to investigate the short term frequency stability and phase- locking performance. It consists A-amplifier; INTintegrator; VCO- voltage controlled oscillator; $\Delta \mathrm{f}$ frequency-phase detector is the output of a frequency to-voltage convertor; $\Delta \phi$-provide linear response to the phase difference between the $\mathrm{rf}$ signal at inputs REF and RF. All rf- and timingsensitive devices are phase locked to a common $10 \mathrm{MHz}$ reference signal. With a frequency- phase detector it was filtered the rf-beat-note signal with a fast tracking oscillator servo bandwidth $1.1 \mathrm{MHz}$ and process the tracking oscillator output signal (8).
Between 50 and $65 \mathrm{MHz}, \Delta \mathrm{f}$ output provides linear frequency to- voltage conversion which is used to generate an error signal for relative frequency stabilization of the two lasers. It is only feedback for the current measurement $\mathrm{f}$ output to the diode laser through the prelock path and drive the acousto-optic modulator with a fixed- frequency signal from a synthesizer with an rf- spectrum analyzer it was simultaneously analyze the beatnote signal directly. Fig. 3 shows the corresponding spectrum of the beat-note signal between two essentially identical diode lasers. We infer a shortterm linewidth of $10 \mathrm{~ms}$ time scale from the 16.1 $\mathrm{kHz}$ of the spectrum of $11.4 \mathrm{kHz}$ for an individual diode laser. The magnitude of the order of 1 to 2 smaller than the typical short- term linewidth of grating stabilized diode laser (9) and emphasizes the excellent short- term stability guaranteed by the new diode laser concept. 


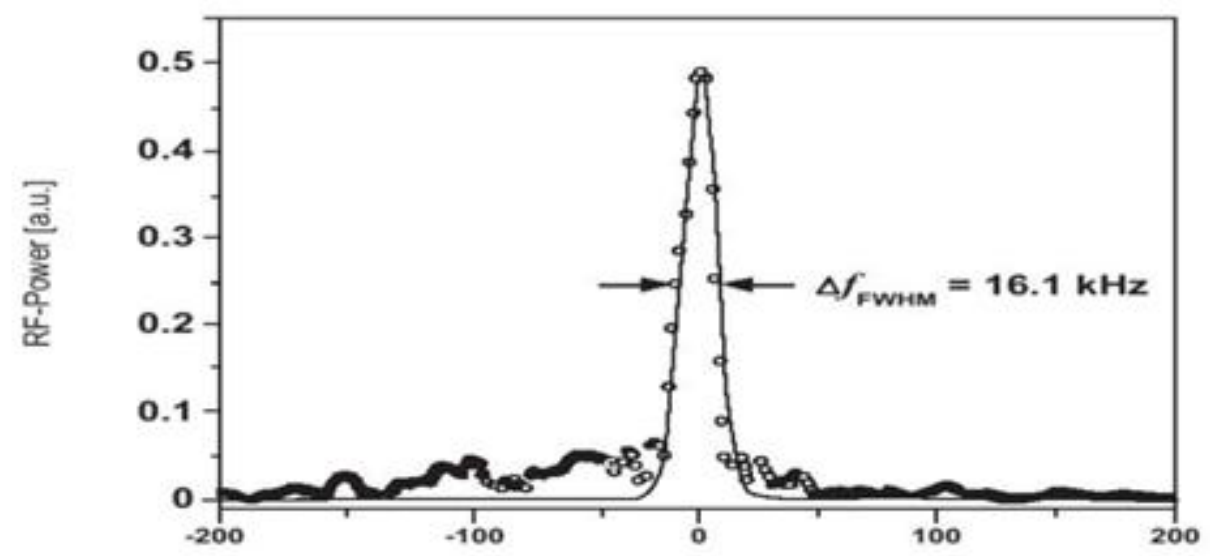

Figure 3. Spectrum of the beat-note signal between two essentially identical diode

Between the two diode lasers and it was next implemented a phase-lock loop and lock the difference frequency to an $\mathrm{rf}$ synthesizer operating at $\mathrm{fRF} \approx 56 \mathrm{MHz}$. At input $\mathrm{REF}$ and $\mathrm{RF}$ with a $0 \ldots \pi$ detection range, the $\Delta \phi$ output of the frequency-phase detector provides a linearized analog measurement of the phase difference between the rf signals (10). To control the phase of a voltage-control oscillator that drives an acoustooptic modulator the phase error signal is filtered and then used. Then it provides fast control of the laser phase (11). The control signal of voltagecontrolled oscillator is further integrated and then fed to a piezo-electric transducer, which setup the actual position of mirror MF of the laser. The low frequency control by this way of the phase-lock loop is provided by the laser so that the acoustooptic modulator is essentially driven at an average frequency that is determined by the frequency the free-running voltage-controlled oscillator.

\section{Results and discussion}

To provide a back- up lock to improve the reliability of the stabilization scheme the $\Delta \mathrm{f}$ output of the frequency-phase detector is still used. By inserting a unity-gain adder into the electronic part of the servo loop next it is determine the phaselock loop servo bandwidth (12). Through the phase- lock loop under closed loop conditions, it is added a perturbation to the loop signal and analyze the propagation of the perturbation. By this way the unity-gain frequency of the servo loop and the open-loop transfer function can be determined. For the open-loop transfer function the measurement reveals a unity-gain frequency of $125 \mathrm{kHz}$.

For the performance of a phase-lock loop a good measurement is the fractional rf power contained within a certain spectral bandwidth at the nominal beat- note carrier frequency. Essentially all power is located at the carrier frequency for sufficiently large spectral bandwidth $>1 \mathrm{MHz}$. The rf power at the carrier frequency drops down unless true phase locking is achieved, which corresponds to a residual broadband rms phase error of significantly less than one rad as the spectral bandwidth is reduced. It is directly recorded beatnote spectra with the rf-spectrum analyzer for various settings of the spectrum analyzer resolution bandwidth between $3 \mathrm{MHz}$ and $1 \mathrm{~Hz}$ to analyze the fractional $\mathrm{rf}$ power contained in the carrier signal (13). It is used an additional $\mathrm{rf}$ synthesizer to realize subhertz resolution bandwidths to mix down the beat note from $56 \mathrm{MHz}$ to $30 \mathrm{kHz}$ and then use a fast Fourier Transform analyzer to record the beat-note spectrum.

\section{Conclusion}

It is demonstrated that the combination of two concepts, the diode laser with resonant optical feedback and the grating diode laser, merges superb short-term frequency stability with excellent tunability and reliability. Linewidth having the short-term of this diode laser system corresponding to $11 \mathrm{kHz}$, and a phase-lock loop servo bandwidth of only $46 \mathrm{kHz}$ is sufficient establish a phase lock between two such diode lasers with subradian residual phase error. Due to excellent performance makes this diode laser concept the first choice for applications that require broadly tunable, ultrastable optical radiation (14). The atomic and molecular coherence experiments are prominent examples which require phase locking between different lasers or between lasers and an optical frequency comb. As part of a flywheel of an optical clock in combination with an ultrastable optical resonator this diode laser could also be used.

\section{Acknowledgement}

We acknowledge the overall assistance received from Indian Institute of Technology, New Delhi during the work. 


\section{References}

1. J.Dostalek et.al, Opt. Lett. 32, 2903 (2007).

2. V.Natasa et.al, Opt. Engg. 51(1), 014201(2012).

3. K. Hashimoto and F. Kannari, Opt. Lett. 17, 2983 (2007).

4. K. Itaya, H. Sugawara, and G. Hatakoshi, J. Cryst, Growth 138, 768 (1994).

5. R.W. Fox, L.W. Gates, and C.W. Hollberg, in Cavity- Enhanced Spectroscopies, R. van Zee and J. Looney, eds. (Academic, 2002), vol.40, pp. 1- 46.

6. K. Doringshoff, I. Ernsting, R.H. Rinkleff, S Schiffer, and A. Wicht, Opt. Lett. 19, 2876 (2007).

7. T.W. Hansch and B. Couilloud, Opt. Commun. 35, 441 (1980).
8. A.D. Ludlow, X. Huang, M. Notcutt, T. Zanon-Willette, S.M. Foreman, M.M. Boyd, S. Blatti and J. Ye, Opt. Lett. 32, 641 (2007).

9. Peik, T. Schneider, and Ch. Tamm, J. Phys. B 39, 145 (2006).

10. R. Wynands and A. Nagel, Appl. Phys. B 68,1 (1999).

11. H. Dongdong, Y.Ling, Opt. Engg. 51(4), 044201(2012).

12. M. Muller, F. Homann, R.H. Rinkleff, A. Wicht, and K. Danzmann, Phys. Rev. A64,013803 (2001).

13. B.M.K Prasad, A. Bawankan, Adb. Mat. \& Res. 1101, 169 ( 2015).

14. H. Loh, Y.J. Lin, I. Teper, M. Cetina, J. Simon, J.K. Thompson, and V. Vuletic, Appl. Opt. 45, 9191 (2006). 